\title{
Utilidad pronóstica de la escala qSOFA en pacientes ingresados en un servicio de Medicina Interna por enfermedades infecciosas
}

\author{
Prognostic utility of the qSOFA scale in patients admitted to an Internal \\ Medicine service due to infectious diseases
}

\begin{abstract}
Ignacio Pérez Catalán', Celia Roig Martí1, Ángela Cubides Montenegro', Alejandro Cardenal Álvarez', Federico Guerrero Jiménez', Paloma Albiol Viñals³ y Jorge Usó Blasco ${ }^{2}$
\end{abstract}

\author{
'Servicio de Medicina Interna, Hospital General Universitario de Castellón, España. \\ ${ }^{2}$ Servicio de Urgencia. \\ ${ }^{3}$ Hospital Clínico de Valencia, España.
}

Los autores declaran no tener conflicto de intereses alguno.

Para la realización del estudio no se ha recibido financiación.

Recibido: 2 de febrero de 2020 (segunda versión: 8 de noviembre de 2020) / Aceptado: 28 de diciembre de 2020

\section{Resumen}

Introducción: La sepsis es una entidad grave siendo su sospecha y tratamiento precoces claves para el pronóstico. Objetivo: Analizar la utilidad pronóstica de la escala qSOFA en pacientes que ingresan por infección en un servicio de Medicina Interna. Pacientes y Métodos: Estudio descriptivo, tranversal, de los pacientes ingresados con infección en el Hospital General de Castellón (España) de noviembre de 2017 a febrero de 2018. Criterio de inclusión: pacientes admitidos por la sospecha de un proceso infeccioso. Variable principal dependiente: mortalidad. Variable principal independiente: qSOFA. Variables secundarias: tiempo hasta primera valoración médica y hasta inicio de antibioterapia empírica en Urgencias (minutos), características demográficas del paciente, analíticas y evolutivas. Resultados: Se analizó un total de 311 pacientes, 145 varones, edad media 78 años (DE 16,23). Setenta y cinco (24\%) presentaron qSOFA $\geq 2$. Se observó una mayor mortalidad en aquellos pacientes con $\mathrm{qSOFA} \geq 2$ (36 vs $11 \%, \mathrm{p}=0,00)$. Conclusión: En pacientes admitidos con enfermedades infecciosas, un valor de qSOFA $>=$ 2 se asoció a mayor mortalidad. Se requieren futuros estudios para comprobar su potencial utilidad diagnóstica.

Palabras clave: sepsis; urgencias; medicina interna; escala SOFA; qSOFA.

\begin{abstract}
Background: Sepsis is a serious entity. Diagnosis and early treatment is important for the prognosis. Aim: To analyze the prognostic utility of the qSOFA scale as a predictor of mortality in patients admitted by infection in an Internal Medicine Service and describe its demographic characteristics and possible association with mortalilty. Methods: Descriptive and cross-sectional study of patients admitted with diagnosis of acute infection at the General Hospital of Castellon (Spain) from November 2017 to February 2018. Inclusion criteria: patients admitted on suspicion of an infectious process. Main dependent variable: mortality. Independent main variable: qSOFA scale. Secondary variables: time until the first medical evaluation and the start of empirical antibiotic therapy, demographic characteristics of the patient, analytics and evolutional. Results: A total of 311 patients were analyzed, 145 men with an average age of 78 (DE 16,23). Seventy five presented qSOFA $\geq 2$. Higher mortality was observed in those patients with qSOFA $\geq 2(36 \%$ vs $11 \%, p=0.00)$. Conclusion: In patients admitted with infectious diseases, a qSOFA value $>=2$ was associated with higher mortality. Future studies are required to verify its potential diagnostic utility.

Keywords: sepsis; emergency care; internal medicine; SOFA score; qSOFA.
\end{abstract}




\section{Introducción}

Tras el III Consenso Internacional de Sepsis (Sepsis-3), se define sepsis como el estado de "disfunción orgánica que amenaza la vida de un paciente, causada por una respuesta anómala del huésped a una infección". Se estima que existen aproximadamente unos 31 millones de casos anuales (19 de ellos graves) con 5 millones de muertes por año, a nivel mundial ${ }^{2}$.

Siendo un cuadro de elevada mortalidad, su detección y tratamiento precoz son claves en el pronóstico, representado por una mortalidad cercana a $50 \%$ a las cuatro horas de haberse instaurado la fase de shock séptico ${ }^{3,4}$.

El consenso Sepsis- $3^{1}$ plantea nuevas definiciones y reemplaza a los criterios del síndrome de respuesta inflamatoria sistémica (SIRS) establecidos tras Sepsis- ${ }^{5}$ y Sepsis-2 $2^{6}$, basándose en algunos criterios clínicos y de laboratorio del Sequential Organ Failure Assessment (SOFA) $\left(\mathrm{PaO}_{2} / \mathrm{FiO}_{2}\right.$, recuento sanguíneo de plaquetas, bilirrubinemia, presión arterial media, puntuación en la escala de Glasgow, creatininemia y diuresis) ${ }^{1}$. Su principal inconveniente es que precisa de los resultados de pruebas de laboratorio para su correcta estimación, por lo que no es una escala óptima a la hora de realizar una detección precoz a la llegada del paciente al servicio de Urgencias. Por ello, en Sepsis-3 se propuso la escala Quick Sequential Organ Failure Assessment (qSOFA) ${ }^{1}$ ya que comprende tres signos clínicos fácilmente valorables en un primer contacto con el paciente incluyendo, durante la atención de urgencias: frecuencia respiratoria $\geq 22 / \mathrm{min}$, presión arterial sistólica $\leq 100 \mathrm{mmHg}$ y alteración del estado mental (Glasgow Coma Scale $<15$ ). La presencia de dos o más de estos criterios tiene un valor predictor similar al del SOFA (AUROC $=0,81 ; 95 \%$ IC 0,80-0,82) e incluso superior en pacientes no UCI, teniendo en cuenta que los pacientes con un SOFA $\geq 2$ presentan una mortalidad durante el ingreso alrededor de $10 \%{ }^{7}$.

De este modo, el qSOFA podría ser una herramienta rápida y simple de aplicar, económica y efectiva para la categorización oportuna de pacientes admitidos por enfermedades infecciosas. Por todo esto, en la actualidad se recomienda el empleo de la escala qSOFA en la categorización de pacientes con sospecha de infección con el fin de priorizar aquellos pacientes más graves (qSOFA $\geq 2)^{1}$. No obstante, se han publicado resultados contradictorios que ponen en duda su capacidad de predecir mortalidad en pacientes de cuidados intensivos ${ }^{1}$ y su sensibilidad para detectar sepsis, en comparación con los clásicos criterios "SIRS"

La población de edad avanzada, cada vez más numerosa en nuestras sociedades, se caracteriza por presentar muchas co-morbilidades, siendo especialmente frágiles ante la enfermedad. Por ello, consideramos necesario el estudio de la utilidad y aplicabilidad de una escala fácil y rápida como es el qSOFA para identificar, ante una enfermedad infecciosa, a esos pacientes con peor pronóstico $\mathrm{y}$, por tanto, con más mortalidad, para poder establecer un manejo precoz y aumentar así la probabilidad de éxito del episodio a tratar.

Por esto, y teniendo en cuenta la escasez de datos en servicios de Medicina Interna, se propone como objetivo general evaluar la utilidad de la puntuación qSOFA como predictor de mortalidad en pacientes que ingresan por enfermedad infecciosa aguda en un servicio de Medicina Interna.

Como objetivos específicos, se planteó determinar qué variables predicen peor pronóstico, con especial interés en el efecto que pueda tener el inicio precoz de la antibioterapia empírica y evaluar la asociación entre qSOFA y estancia hospitalaria

\section{Pacientes y Métodos}

Estudio descriptivo y transversal de los pacientes ingresados con diagnóstico de infección en Medicina Interna del Hospital General de Castellón (España) desde noviembre de 2017 hasta febrero de 2018.

\section{Criterio de inclusión}

Cualquier paciente $>15$ años, ingresado en el Servicio de Medicina Interna con sospecha de patología infecciosa.

\section{Criterio de exclusión}

Pacientes que no contaban con registros clínicos completos.

Variable principal independiente: la puntuación qSOFA. Variable principal dependiente: la mortalidad durante el ingreso. Variables secundarias: el tiempo desde categorización en Urgencias hasta la primera valoración médica y hasta el inicio de la antibioterapia empírica en Urgencias (minutos), características demográficas y antecedentes del paciente, resultados de estudio microbiológico (incluye: hemocultivos, cultivo de esputo, serologías según sospecha clínica, reacción de polimerasa en cadena [RPC] en hisopado nasofaríngeo para influenza A/B y virus respiratorio sincicial, antigenuria de Legionella pneumophila y Streptococcus pneumoniae, urocultivo), el foco de infección según la impresión diagnóstica del médico responsable, el valor de proteína C-reactiva-PCR (valores de referencia $3-10 \mathrm{mg} / \mathrm{L}$ ) y procalcitonina (valores de referencia $0,00-0,10 \mathrm{ng} / \mathrm{mL}$ ) al ingreso, la presencia de bacteriemia, la estadía hospitalaria, el ingreso en UCI o no y la mortalidad hospitalaria.

La categorización (triage) en urgencias se realizó según el sistema de priorización de Manchester, mediante un sistema informático con preguntas estandarizadas, el 
cual le asigna al paciente un nivel de prioridad de atención médica ${ }^{9}$.

Se recogió la información en un registro escrito de datos, sobre todas las variables analizadas, como paso previo a la elaboración de una base de datos informática y anonimizada. Para ello, se obtuvo la información de la historia electrónica hospitalaria, tanto la puntuación qSOFA calculada por el médico de Urgencias que valoró inicialmente al paciente en un primer contacto, como los resultados de las pruebas complementarias y la evolución posterior del paciente.

Se realizó test de $\chi^{2}$ para variables cualitativas y U de Mann Whitney para variables cuantitativas. Finalmente, se realizó análisis multivariado mediante regresión lineal. Se asumió significación estadística si $\mathrm{p}<0,05$, con un IC $95 \%$. Los datos fueron procesados haciendo uso del programa informático SPSS Statistics 23.

El estudio fue aprobado por el Comité de Ética e Investigación Clínica del centro. Los procedimientos seguidos se conformaron a las normas éticas del comité de experimentación humana responsable y de acuerdo con la Asociación Médica Mundial y la Declaración de Helsinki.

\section{Resultados}

Un total de 311 pacientes fueron incluidos en el estudio, 145 varones $(46,7 \%)$, con una edad media de 78 años (DE 16,23) (83,5\% mayores de 65 años). De ellos, 83 con enfermedad renal crónica $(26,6 \%), 80$ diabéticos $(25,8 \%), 16$ con tratamiento inmunosupresor, ya fuese corticoterapia, terapia inmunosupresora o fármacos biológicos $(5,1 \%), 7$ con neoplasias hematológicas $(2,2 \%)$ y 6 con cirrosis hepática $(1,9 \%)$.

Tiempo medio transcurrido hasta primera valoración médica en Urgencias: 128 min (DE 115,59) y hasta inicio de antibioterapia empírica: 315 min (DE 200,04) (Tabla 1).

Se extrajeron 200 pares de hemocultivos (29 casos de bacteriemia) y en 187 pacientes se administró antibioterapia empírica, siendo levofloxacina el más frecuente (38,8\%).

El foco infeccioso más frecuente fue el respiratorio (70,8\%), seguido del urinario $(13,8 \%)$.

Los valores medios de PCR y procalcitonina fueron $166 \mathrm{mg} / \mathrm{L}$ (DE $142 \mathrm{mg} / \mathrm{L}$ ) y 5,96 ng/mL (DE 16,33 ng/ $\mathrm{mL})$, respectivamente. Se obtuvo valores de PCR de todos los pacientes y de procalcitonina de 239 pacientes (76,8\%).

Ciento cuarenta y dos pacientes presentaron aislamiento microbiológico $(45,6 \%)$, siendo el más frecuente Escherichia coli $(29,6 \%)$ y 16 de los 142 fueron microorganismos multi-resistentes (con mayor frecuencia Staphylococcus aureus resistente a meticilína y E. coli
Tabla 1. Pacientes ingresados en el Servicio de Medicina Interna con sospecha de patología infecciosa. Descripción de la muestra

n (\%)

Totalidad de la muestra

$311(100)$

Edad media (años)

78

Sexo (varones)

78 (25)

Enfermedad renal crónica

$83(26,7)$

Diabetes mellitus

$80(25,6)$

Tratamiento inmunosupresor

$16(5,1)$

Neoplasia hematológica

$7 \quad(2,2)$

Cirrosis hepática

$6(1,9)$

Hemocultivos extraídos

$200 \quad(64)$

Hemocultivos positivos

$29(14,5)$

Tiempo medio hasta $1^{\text {a }}$ valoración médica

(minutos)

128

(DE 115,59)

Inicio de antibioterapia empírica en Urgencias (2 más frecuentes)

$187(59,9)$

Levofloxacina

Ceftriaxona

Tiempo medio hasta inicio de antibioterapia empírica

(minutos)

(DE 200,04)

Aislamiento microbiológico

142

Acierto de antibioterapia según antibiograma de aislamiento microbiológico posterior

Foco de origen infeccioso (2 más frecuentes)

Respiratorio

$221(70,7)$

Urinario

$43(13,7)$

Valor medio de PCR

166

(mg/L)

(DE 142)

Valor medio de procalcitonina

(ng/mL)

5,96

(DE 16,33)

Estancia (rango; mediana) (días)

$1-42 ; 7$

Ingreso en $\mathrm{UCl}$

$4 \quad(1,28)$

Mortalidad

$53(17)$

productora de BLEE con 8 y 6 casos, respectivamente). Se obtuvieron además 24 RPC positivas de influenza A y 14 de influenza B. De los 142, recibieron antibioterapia empírica en Urgencias 96, siendo acertada según antibiograma posterior en 60 de ellos $(62,5 \%)$.

La estadía media fue de 8,3 días (1-42; DE 5,43), 4 pacientes precisaron ingreso en UCI y la mortalidad global fue de $17 \%$. El estudio descriptivo de la muestra queda resumido en la Tabla 1.

Del total de pacientes, 75 (24\%) presentaron a su llegada $q S O F A \geq 2$. El tiempo medio hasta primera valoración médica en Urgencias para este grupo fue significativamente menor en comparación con el resto, siendo éstos de 93 y 139 min, respectivamente $(\mathrm{p}=0,001)$; en cambio, 


\begin{tabular}{|c|c|c|c|c|c|c|}
\hline Variables cualitativas & $\mathbf{n}$ & $\chi^{2}$ & p & Variables cuantitativas & & $\begin{array}{l}\text { ficación U de } \\
\text { nn Whitney }\end{array}$ \\
\hline Sexo (varones) & 145 & 0,671 & 0,413 & Edad media (años) & 78 & 0,254 \\
\hline Insuficiencia renal & 83 & 0,958 & 0,619 & Tiempo hasta valoración médica (min) & 128 & 0,150 \\
\hline Diabetes mellitus & 80 & 0,318 & 0,573 & Tiempo hasta antibioterapia (min) & 315 & 0,456 \\
\hline Tratamiento inmunosupresor & 16 & 0,221 & 0,638 & PCR media (mg/L) & 166 & 0,016 \\
\hline Patología hematológica & 7 & 1,471 & 0,225 & Procalcitonina media $(\mathrm{ng} / \mathrm{mL})$ & 5,96 & 0,237 \\
\hline Cirrosis hepática & 6 & 4,701 & 0,03 & & & \\
\hline Administración de antibioterapia en Urgencias & 186 & 1,595 & 0,207 & \multicolumn{3}{|c|}{ Tabla 3. Análisis multivariado de la asociación con mortalidad } \\
\hline qSOFA $\geq 2$ & 75 & 24,943 & $0,5 \times 10^{-6}$ & \multirow{2}{*}{$\begin{array}{l}\text { Variables } \\
\text { qSOFA } \geq 2\end{array}$} & \multicolumn{2}{|c|}{ Significación (p) } \\
\hline Código Sepsis & 33 & 0,645 & 0,422 & & \multicolumn{2}{|c|}{$0,5 \times 10-5$} \\
\hline $\begin{array}{l}\text { Tipo de infección } \\
\text { Respiratoria }\end{array}$ & 221 & $\begin{array}{c}14,293 \\
0,306\end{array}$ & $\begin{array}{l}0,046 \\
0,580\end{array}$ & Cirrosis hepática & \multicolumn{2}{|c|}{0,028} \\
\hline Urinaria & 43 & 1,035 & 0,309 & $\mathrm{PCR}$ & \multicolumn{2}{|c|}{0,03} \\
\hline Aislamiento microbiológico & 142 & 0,338 & 0,561 & Tipo de infección & \multicolumn{2}{|c|}{0,07} \\
\hline Acierto en la antibioterapia & 60 & 0,180 & 0,672 & & & \\
\hline
\end{tabular}

no hubo diferencias estadísticamente significativas en el tiempo medio de inicio de la antibioterapia empírica, siendo éstos de 299 (en 57 casos) y 322 (en 130 casos) minutos, respectivamente $(\mathrm{p}=0,198)$.

En cuanto al objetivo principal, los pacientes que a su llegada a Urgencias presentaron qSOFA $\geq 2$ mostraron una mortalidad de $36 \%$ frente al resto que fue de $11 \%$, siendo la diferencia estadísticamente significativa $(\mathrm{p}=0,5$ x $10^{-6}$ ).

Por otra parte, de todas las variables analizadas en el estudio, se relacionaron de forma estadísticamente significativa con mortalidad la cirrosis hepática, la obtención de qSOFA $\geq 2$ a su llegada, valores elevados de PCR y también variaba la mortalidad según fuera el foco de la infección (mayor mortalidad si el origen era una úlcera por presión sobre-infectada $\mathrm{y}$, en segundo lugar, si era abdominal) (Tabla 2). Los valores de procalcitonina y el inicio precoz de la antibioterapia empírica no influyeron en la mortalidad. Se analizaron de forma separada los pacientes que recibieron una antibioterapia adecuada y en este subgrupo el tiempo hasta el inicio de ésta tampoco se asoció con la mortalidad.

El análisis multivariado reveló que, tanto el presentar qSOFA $\geq 2\left(\mathrm{p}=0,5 \times 10^{-5}\right)$, valores elevados de PCR $(p=0,03)$ y el antecedente de cirrosis hepática $(p=0,028)$, se asociaron en forma independiente a mortalidad. En cambio, el tipo de foco infeccioso no reveló ser factor independiente (Tabla 3).

No se observó asociación entre las diferentes puntuaciones de qSOFA y estadía hospitalaria $(\mathrm{p}=0,596)$.

\section{Discusión}

La sepsis supone un riesgo vital para los pacientes; su reconocimiento y tratamiento precoces resultan clave para mejorar su pronóstico. En este sentido, la escala qSOFA ha demostrado ser una herramienta útil, sobre todo por su sencillez, rapidez y disponibilidad en un primer contacto con el paciente. Permite seleccionar a aquellos pacientes que tienen una mayor probabilidad de fallecer. Existen múltiples estudios que avalan su validez, tanto en pacientes ingresados en Unidades de Cuidados Intensivos ${ }^{10}$ como en pacientes quirúrgicos, pero existe poca evidencia en pacientes ingresados en un servicio de Medicina Interna. Además, dichos pacientes como podemos observar en el estudio, son generalmente pacientes de edad avanzada, con múltiples co-morbilidades, y es sabido que tanto las características biológicas como clínicas de los pacientes influyen en el pronóstico del proceso séptico (éste probablemente sea el motivo de la elevada mortalidad observada en el estudio y el escaso número de ingresos en UCI al no ser subsidiarios la gran mayoría de medidas invasivas) ${ }^{11}$. No obstante, se observa una clara asociación del valor del qSOFA presentado a su llegada a Urgencias con mortalidad durante el ingreso, siendo ésta para valores de qSOFA $\geq 2$ más del triple del $10 \%$ estimado en Sepsis- $3^{7}$.

Por otra parte, de manera estadísticamente significativa, estos pacientes recibieron una primera valoración médica más precoz, pero esto no supuso un inicio más temprano de la antibioterapia empírica. Ha de tenerse en 
cuenta que en nuestro centro se usa el sistema de categorización de Manchester y que no se utiliza de forma sistemática el qSOFA. Además, en aquellos pacientes con sospecha de proceso infeccioso que según el sistema de Manchester presentan especial gravedad simplemente se prioriza su asistencia sin existir protocolo especial alguno, bundle o equipo de sepsis, pudiéndose explicar así estos hallazgos. No obstante, como ya se ha comentado, se trata de una muestra con elevada co-morbilidad, en la cual en ocasiones pueden existir procesos infecciosos que se presentan de manera inusual, dificultando así el diagnóstico diferencial y, por tanto, retrasando el inicio de la antibioterapia empírica.

Únicamente qSOFA $\geq 2$, valores elevados de PCR y el antecedente de cirrosis hepática fueron capaces de predecir mayor mortalidad. Los valores elevados de procalcitonina no permitieron predecir peor pronóstico, en contra de lo observado en anteriores estudios ${ }^{12,13}$. Estos resultados hacen aún más necesario e interesante el empleo precoz de la puntuación qSOFA ante la sospecha de una patología infecciosa en unidades no críticas.

El inicio de antibioterapia empírica en Urgencias no se relacionó con disminución de la mortalidad, ni su precocidad y ni siquiera su acierto en relación a aislamiento microbiológico posterior en los cultivos extraídos al ingreso. Estos resultados van en contra de muchas publicaciones clásicas y más recientes en las que se objetiva una mejoría clara del pronóstico en aquellos pacientes que reciben antibioterapia empírica precoz ${ }^{3,4,14}$. No obstante, existe debate en la actualidad sobre si verdaderamente todos los pacientes con proceso infeccioso verían beneficiado su pronóstico con el inicio precoz de la antibioterapia. De hecho, existen publicaciones que defienden que aquellos pacientes que ciertamente se beneficiarían serían exclusivamente aquellos en shock séptico o compromiso hemodinámico ${ }^{15-17}$.
Como limitaciones, en nuestro estudio no ha sido posible categorizar a los pacientes según tuvieran un proceso infeccioso leve, tuvieran sepsis o en cambio presentaran shock séptico, por lo que no podemos aportar más sobre esta última cuestión, pero podría ser objeto de análisis en futuras investigaciones. Tampoco se recogen otras intervenciones que pudieran influir en la mortalidad como podría ser la fluidoterapia y el control precoz del foco infeccioso. Además, cabe añadir que contamos con un pequeño tamaño muestral en comparación con estudios similares y que los tiempos tanto de primera valoración médica en Urgencias como de inicio de la antibioterapia empírica son los registrados en la historia electrónica del paciente y en la aplicación de prescripción electrónica del centro, habiendo podido sufrir en la práctica ciertas variaciones. Por último, probablemente los resultados también pudieran estar influidos por co-morbilidades importantes no analizadas que puedan sufrir nuestros pacientes.

\section{Conclusión}

Según los resultados de nuestro estudio, existe aparente asociación entre qSOFA y mortalidad y no se observó impacto de la precocidad de la terapia antimicrobiana. Sin embargo, dada las importantes limitaciones metodológicas señaladas, se requieren futuros estudios para responder estos relevantes interrogantes.

Agradecimientos. A todo el Servicio de Urgencias Generales del Hospital General de Castellón, así como al Servicio de Medicina Interna y en concreto a la Unidad de Enfermedades Infecciosas por su inestimable ayuda durante el desarrollo de este estudio, sin los cuales no hubiera sido posible.

\section{Referencias bibliográficas}

1.- Singer M, Deutschman C S, Seymour C W, Shankar-Hari M, Annane D, Michael Bauer $\mathrm{M}$, et al. The Third International Consensus Definitions for Sepsis and Septic Shock. JAMA. 2016; 315 (8): 801-10. doi: 10.1001/ jama.2016.0287.

2.- Fleischmann C, Scherag A, Adhikari N K, Hartog C S, Tsaganos T, Schlattmann P, et al. International Forum of Acute Care Trialists. Assessment of global incidence and mortality of hospital-treated sepsis: current estimates and limitations. Am J Respir Crit Care Med. 2015 193 (3): 259-72. doi:10.1164/rccm.2015040781OC.

3.- Kumar A, Roberts D, Wood K E, Light B,
Parrillo J E, Sharma S, et al. Duration of hypotension before initiation of effective antimicrobial therapy is the critical determinant of survival in human septic shock. Crit Care Med. 2006; 34 (6): 1589-96. doi: 10.1097/01. CCM.0000217961.75225.E9.

4.- Kumar A. Optimizing antimicrobial therapy in sepsis and septic shock. Crit Care Clin. 2009; 25 (4): 733-51. doi:10.1016/j. ccc.2009.08.004

5.- Bone R C, Balk R A, Cerra F B, Dellinger R P, Fein A M, Knaus W A, et al. Definitions for sepsis and organ failure and guidelines for the use of innovative therapies in sepsis. The ACCP/SCCM Consensus Conference Committee. American College of Chest Physicians/ Socie ty of Critical Care Medicine.
Chest. 1992; 101 (6): 1644-55. doi: 10.1378/ chest.101.6.1644.

6.- Levy M M, Fink M P, Marshall J C, Abraham E, Angus D, Cook D, et al. 2001 SCCM/ ESICM/ACCP/ATS/SIS International Sepsis Definitions Conference. Intensive Care Med. 2003; 29 (4): 530-8. doi: 10.1007/s00134-0031662-x.

7.- $\quad$ Seymour C W, Liu V, Iwashyna T J, Brunkhorst F M, Rea T D, Scherag A, et al. Assessment of clinical criteria for sepsis. JAMA. 2016; 315 (8): 762-774. doi:10.1001/jama.2016.0288.

8.- Serafim R, Gomes J A, Salluh J, Póvoa P. A comparison of the Quick-SOFA and systemic inflammatory response syndrome criteria for the diagnosis of sepsis and prediction of mortality: a systematic review and meta- 
analysis. Chest. 2018; 153 (3) : 646-55. doi:10.1016/j.chest.2017.12.015.

9.- Mackway-Jones K, Marsen J, Windle J. eds. Emergency Triage: Manchester Triage Group. London: BMJ Publishing Group; 1997.

10.- Vincent J L, de Mendonça A, Cantraine F, Moreno R, Takala J, Suter P M, et al. Use of the SOFA score to assess the incidence of organ dysfunction/failure in intensive care units: results of a multicenter, prospective study. Working Group on "Sepsis-Related Problems" of the European Society of Intensive Care Medicine. Crit Care Med. 1998; 26 (11): 1793-800. doi: 10.1097/00003246-19981100000016.

11.- Iskander K N, Osuchowski M F, StearnsKurosawa D J, Kurosawa S, Stepien $\mathrm{D}$, Valentine $\mathrm{C}$, et al. Sepsis: multiple abnormalities, heterogeneous responses, and evolving understanding. Physiol Rev. 2013; 93 (3): 1247-88. doi:10.1152/physrev.00037.2012.

12.- Uzzan B, Cohen R, Nicolas P, Cucherat M, Perret G Y. Procalcitonin as a diagnostic test for sepsis in critically ill adults and after surgery or trauma: a systematic review and metaanalysis. Crit Care Med. 2006, 34: 1996-2003. doi:10.1097/01.CCM.0000226413.54364.36.

13.- Sanjeev Sinha S J, Sharma S K, Samantaray J C, Aggrawal P, Vikram N K, et al. Procalcitonin as a prognostic marker for sepsis: a prospective observational study. BMC Res Notes 2014; 7 : 458. doi: 10.1186/1756-0500-7-458.

14.- Seymour C W, Gesten F, Prescott H C, Friedric M E, Iwashyna T J, Gary S. Phillips G S, et al. Time to treatment and mortality during mandated emergency care for sepsis. N Engl J Med. 2017; 376 (23): 2235-44. doi 10.1056/ NEJMoa1703058.
15.- Klompas M, Calandra T, Singer M. Antibiotics for sepsis-finding the equilibrium. JAMA. 2018; 320 (14): 1433-4. doi: 10.1001/ jama.2018.12179.

16.- Liu V X, Fielding-Singh V, Greene J D, Baker $\mathrm{J} \mathrm{M}$, Iwashyna $\mathrm{T} \mathrm{J}$, Bhattacharya $\mathrm{J}$, et al. The timing of early antibiotics and hospital mortality in sepsis. Am J Respir Crit Care Med. 2017; 196 (7): 856-63. doi 10.1164/ rccm.201609-1848OC.

17.- Alam N, Oskam E, Stassen P M, Exter P V, van de Ven P M, Haak H R, et al; PHANTASi Trial Investigators and the ORCA (Onderzoeks Consortium Acute Geneeskunde) Research Consortium the Netherlands. Prehospital antibiotics in the ambulance for sepsis: a multicentre, open label, randomised trial. Lancet Respir Med. 2018; 6 (1): 40-50. doi:10.1016/S2213-2600(17)30469-1. 